in clinical allergy and immunology and has recently recognised a training course in internal medicine for this specialty. The National Health Service must face up to this problem as already many sufferers from allergy are resorting to fringe medicine.

In conclusion, careful control of hyposensitisation is required. Only insect venoms, grass pollens, and perhaps house dust mite vaccines should be used. The modern vaccines are potent, containing highly purified antigen, and therefore great care is required in using this treatment, particularly for patients with asthma. Full facilities for cardiopulmonary resuscitation must be immediately available, and patients must be carefully monitored before, during, and (at least for now) for two hours after injections. A review of hyposensitisation deaths along the lines of the British Thoracic Society investigation of asthma deaths would be valuable. Finally, medical training in clinical allergy and immunology should be urgently improved so that this specialty can be expanded in the National Health Service.

J O WARNER Consultant paediatric chest physician

Brompton Hospital,

London SW3 6HP

JAMES W KERR

Consultant physician in respiratory diseases

Western Infirmary,

Glasgow G11 6NT

1 Committee on Safety of Medicines. CSM Update: Desensitising vaccines. BrMed $\mathcal{f}$ 1986;293:148.

2 Noon L. Prophylactic inoculation for hay fever. Lancet $1911 ;$ i:1572.

3 Freeman J. Further observations on the treatment of hay fever by hypodermic inoculations of pollen vaccine. Lancet $1911 ;$;i:814.

4 Hunt KJ, Valentine MD, Sobotka AK, Benton AW, Amodis FJ, Lichtenstein LM. A controlled trial of immunotherapy in insect hypersensitivity. N Engl f Med 1978;299:257-61.

5 Lichtenstein LM, Norman PJ, Winkenwerder WL. A single year of immunotherapy for ragweed

hay fever. Ann Intem Med 1971;75:663-71.
6 Frankland AW, Augustin R. Prophylaxis of summer hay fever and asthma. Lancet 1954;i:1055-7.

6 Frankland AW, Augustin R. Prophylaxis of summer hay fever and asthma.

7 Ewan PW. Allergy to insect stings: a review. $\mathcal{F} R$ Soc Med 1985;78:234-9.

8 Mygind N. Nasal allergy. Oxford: Blackwell Scientific Publications, 1978.

Warner JO, Price JF, Soothill JF, Hey EN. Controlled trial of hyposensitisation to Dermatophagoides pteronyssinus in children with asthma. Lancet 1978;ii:912-5.

10 Warner JO. Hyposensitisation in asthma: a review. $\mathcal{F}$ Soc Med 1981;74:60-5.

11 Vervloet D, Khairallah E, Arnaud A, Charpin JA. A prospective national study of the safety of immunotherapy. Clin Allergy 1980;10:59.

\section{Doctors and the death penalty: an international issue}

One of the recent features in Britain has been the regular attempts in parliament to have capital punishment restored. It is an argument that has particular relevance for doctors. The introduction of execution by lethal injection in several states in the United States has caused concern among doctors, most of whom are disturbed that drugs and practices developed for treatment are being used to kill. Curran and Casscells concluded in $\mathbf{1 9 8 0}$ that for doctors to participate in execution by lethal injection would be contrary to medical ethics. ${ }^{1}$ But even when doctors are not giving the injections they may be required to determine the physical and mental fitness of prisoners for execution, provide technical advice, prescribe the drugs, supervise their administration, or examine the prisoner during the execution so that it can continue if he is not yet dead. ${ }^{2}$ In 1977, for example, Oklahoma required doctors to supervise the execution process but dropped this requirement after several American state medical associations declared that direct participation by doctors in lethal injections was ethically unacceptable. In 1980 the American Medical Association as a whole adopted a resolution that stated that "a physician, as a member of a profession dedicated to the preservation of life when there is $c_{c}$ hope of doing so, should not be a participant in a legally authorised execution."

A recent report by Amnesty International on the deatho penalty in the US raises uncomfortable issues for doctors. ${ }^{2}$ Ino one infamous case in 1977 the medical director of the Texas Department of Corrections checked that a convicted murderer's veins were suitable for injection, provided the medical technicians who gave the lethal dose with the drug, supervised them, and examined the man on several occasions to see if he was dead. The electrocution of a murderer in Alabama in 1983 needed three separate jolts of 1900 volts overo 14 minutes before the supervising doctors could pronounces that the prisoner was dead. During the first jolt the electrode $\vec{\circ}$ on the condemned man's leg burnt through and fell off. $\rightarrow$ During the second jolt smoke and flames erupted from his $\vec{\rho}$ left temple and leg. An execution by electrocution in Georgiao in 1984 needed two shocks, and it took six minutes after the first charge for the body to cool enough before doctors couldo examine it. The prisoner took 23 breaths, and the two doctors stated that he was still alive. Ten minutes after the first charge the second and fatal charge was given. None of these examples provide much support for the argument thatoo death by injection and electrocution represent a humane advance over death by hanging.

More important is the dilemma facing those doctors $\$$ attending bungled executions: the person they examined was alive, but they were required by the state not to sustain his life. On the contrary, they were implicitly required to indicate to the executioner that the man required more trauma to complete the execution. This seems to be in conflict with medical ethics and to suggest that the doctors were doing more than what is permitted by the Worldo Medical Association's 1981 declaration that "a physician's only role would be to certify death once the state had carried? out the execution."

The Amnesty report also draws attention to the ethica dilemmas faced by psychiatrists looking after condemned? but psychotic patients. It is a civilised ethical principle that insane prisoners should not be executed (although thes reintroduction of capital punishment in the US has producedo some appalling miscarriages of justice and ethical practice) but in some states this merely means that the psychiatrists are? required to treat the mental illness so that with his mental health restored the condemned prisoner can be executed.? Not surprisingly, the American Psychiatric Association haso condemned this as "a perversion of medical ethics" and has opposed psychiatrists participating in capital punishment. ${ }^{3}$.

Elsewhere in the world the death penalty has beeno discussed within professional associations. The Secretary General of the Brazil Medical Association recently arguede that "the doctor's role is to alleviate pain and to prolonge life... Doctors can never, under any circumstances, be incs favour of the death penalty ... Those who execute should assume full responsibility; doctors should have no part ino this."'4 He went on to suggest that "this be policy of medica $\mathbb{R}$ bodies world wide." In June 1986 the medical associations of the Nordic countries (Denmark, Finland, Iceland, Norway and Sweden) resolved that it is "indefensible for anyo physician to participate in any act connected to and necessarye for the administration of capital punishment." In Britain doctors have been silent recently on the death penalty, 
probably because its reintroduction becomes ever more unlikely. Nevertheless, the ethical issues raised by the Amnesty report concern doctors throughout the world and not merely those in the United States. Doctors have an important part to play in abolishing what is cruel, inhuman, and degrading punishment. Firstly, they must articulate and implement ethical codes that unambiguously prohibit doctors participating in executions, and, secondly, they must widen the discussion to include the broader ethical issues of the death penalty.

ANTHONY W ClARE

Professor of Psychological Medicine,

St Bartholomew's Hospital Medical College,

London EC1A 7BE

1 Curran WJ, Casscells W. The ethics of medical participation in capital punishment by intravenous drug injection. $N$ Engl I Med 1980;302:226-30.

2 Amnesty International. United States of America: the death penaly. London: Amnesty International Publications, 1987.

3 Casscells W, Curran WJ. Doctors, the death penalty and lethal injections. N Engl 7 Med 1982;307:1532-3.

4 Amnesty International. The death penaly in the United States: an issue for health professionals. London: Amnesty International Publications, 1987:13. (Quotation from fornal da Associacao Medica Brasileira 1985 November:12.)

\section{Penetration of antibiotics into the respiratory tract}

Few antibiotics penetrate well into broncial secretions, and yet most respiratory infections respond to treatment. The poor penetration seems to matter only in patients with chronic suppurative airways disease (bronchitis, bronchiectasis, and cystic fibrosis) and in those infected with less sensitive organisms and may then contribute to recurrent sepsis. In these patients the initial response to antibiotics may be good because the drugs penetrate the mucosa better than the secretions, but subtherapeutic concentrations in the mucus may lead to relapse. Furthermore, penetration may become worse as tissue damage progresses.' For these reasons doctors, and particularly those looking after patients with chronic suppurative lung diseases, need to know something about the penetration of antibiotics into the respiratory tract.

The penetration of $\beta$ lactam antibiotics is modest-peak sputum concentrations of penicillins are only $5-20 \%$ of those in serum. Even $1 \mathrm{~g}$ of oral ampicillin will not always attain inhibitory concentrations for haemophilus, although activity against more sensitive bacteria such as Streptococcus pneumoniae is readily achieved. ${ }^{2}$ Parenteral ampicillin gives higher concentrations in both the serum and sputum. Amoxycillin is more completely absorbed from the gastrointestinal tract and achieves higher serum and sputum concentrations than oral ampicillin, ${ }^{34}$ though even $750 \mathrm{mg}$ will not always produce inhibitory sputum activity against haemophilus. ${ }^{5}$ Cole and colleagues have reported longer remissions in patients with chronic bronchitis after short courses of high dose oral amoxycillin ( $3 \mathrm{~g} 12$ hourly), though peak sputum concentrations varied widely. ${ }^{6}$ Davies and Maesen found higher sputum ampicillin concentrations after bacampicillin $(800 \mathrm{mg})$ than after ampicillin $(1 \mathrm{~g}){ }^{5}$ although eight hourly $400 \mathrm{mg}$ or $800 \mathrm{mg}$ doses of bacampicillin controlled haemophilus infections in bronchitis. ${ }^{7}$ Standard oral doses of cloxacillin in patients with cystic fibrosis scarcely exceed inhibitory concentrations for Staphylococcus aureus. ${ }^{8}$ Broad spectrum penicillins such as carbenicillin, piperacillin, ticarcillin, and mezlocillin do not always produce adequate activity against pseudomonas in respiratory secretions, ${ }^{9-11}$ and they are best used with an aminoglycoside in patients with serious infection. Cephalexin is active against Str pneumoniae in sputum, but the concentrations are not likely to be inhibitory for Haemophilus influenzae. ${ }^{12}$ Injectable cephalosporins such as cefuroxime, cefazolin, and cefotaxime achieve higher serum concentrations, and peak concentrations in sputum are at least four times higher than those resulting from oral agents. ${ }^{1013}$

Erythromycin is widely used in treating respiratory infections because of its activity against mycoplasma, legionella, and various other bacteria-pneumococci and branhamella are very susceptible, but concentrations needed to inhibit $H$ influenzae are higher. Erythromycin produces good but variable sputum concentrations when given intravenously ${ }^{14}$ though much lower concentrations (which may be subinhibitory for haemophilus) after a $500 \mathrm{mg}$ oral dose. ${ }^{15}$ Much greater activity is detected in lung tissue after oral and intravenous erythromycin. Clindamycin and rifampicin readily attain good sputum activity against Staph aureus, ${ }^{10}$ and rifampicin is a suitable adjunct to antibiotics such as flucloxacillin or vancomycin in staphylococcal pneumonia. Anaerobes (implicated in aspiration pneumonia) are inhibited by bronchial concentrations of metronidazole after $400 \mathrm{mg}$ oral doses. ${ }^{16}$

Tetracycline concentrations in bronchial secretions are inhibitory to most strains of Str pneumoniae, though activity against $H$ influenzae is not always adequate. ${ }^{17}$ Sputum antibiotic concentrations and clinical results may correlate poorly: Maesen and colleagues found that haemophilus strains with minimum inhibitory concentrations of doxycycline exceeding $2 \mathrm{mg} / 1$ were rarely eradicated by conventional doses of doxycycline in patients with chronic bronchitis, although almost two thirds of isolates with lower minimum inhibitory concentrations responded, despite a mean peak sputum concentration of only $0.3 \mathrm{mg} / 1 .^{18}$ Considerably higher doxycycline concentrations are, however, reached in the bronchial wall and lung tissue. ${ }^{19}$

Treatment of respiratory infections caused by Gram negative organisms with gentamicin is most likely to succeed if peak serum concentrations exceed $8 \mathrm{mg} / 1 .{ }^{20}$ Studies in dogs have shown that peak concentrations in bronchial mucus are about one quarter of those in the serum and that even high doses of gentamicin may fail to reach therapeutic concentrations against Pseudomonus aeruginosa in respiratory secretions. ${ }^{21}$ Adequate concentrations may not be readily achieved in the elderly or in patients with renal impairment, when dosage must be carefully controlled to avoid toxicity: combination with a broad spectrum penicillin may produce synergy, but newer antipseudomonal agents such as ceftazidime are safer.

Trimethoprim passes readily into bronchial secretions, and concentrations often exceed those in serum, ${ }^{22}$ though sulphamethoxazole activity after oral co-trimoxazole may be subtherapeutic. Brumfitt and colleagues detected no sulphamethoxazole and variable trimethoprim concentrations in sputum in 24 patients given co-trimoxazole, though both drugs were equally effective clinically. ${ }^{23}$ Quinolones have renewed interest in antibiotic pharmacokinetics in the lung because, despite effective diffusion in bronchi and good antimicrobial activity including against haemophilus and branhamella, they are only moderately active against Str pneumoniae. Peak sputum concentrations exceed half of those 